\title{
Hardness-Based Plasticity and Fracture Model for Quench- Hardenable Boron Steel (22MnB5)
}

\author{
L. Greve $\mathrm{a}^{\mathrm{a}}$, T. K. Eller ${ }^{\mathrm{a}, \mathrm{b}}$, M. Medricky ${ }^{\mathrm{a}}$, M. Andres ${ }^{\mathrm{a}}$ \\ ${ }^{a}$ Volkswagen AG, Letter Box 1777, Wolfsburg, 38436, Germany, lars.greve@volkswagen.de, \\ tom.karl.eller@volkswagen.de,miloslav.medricky@volkswagen.de, michael.andres@volkswagen.de \\ ${ }^{b}$ University of Twente, P.O. Box 217, 7500 AE Enschede, The Netherlands
}

\begin{abstract}
A comprehensive strain hardening and fracture characterization of different grades of boron steel blanks has been performed, providing the foundation for the implementation into the modular material model (MMM) framework developed by Volkswagen Group Research for an explicit crash code. Due to the introduction of hardness-based interpolation rules for the characterized main grades, the hardening and fracture behavior is solely described by the underlying Vickers hardness. In other words, knowledge of the hardness distribution within a hot-formed component is enough to set up the newly developed computational model. The hardness distribution can be easily introduced via an experimentally measured hardness curve or via hardness mapping from a corresponding hot-forming simulation. For industrial application using rather coarse and computationally inexpensive shell element meshes, the user material model has been extended by a necking/post-necking model with reduced mesh-dependency as an additional failure mode. The present paper mainly addresses the necking/post-necking model.
\end{abstract}

Keywords: Tailored properties, Hot forming, Hardness, Fracture modeling, Mohr-Coulomb, 22MnB5 PACS: 46.35.+z, 46.50.+a, 46.70.-p, 46.80.+j

\section{INTRODUCTION}

Three sets of 22MnB5 sheets (commercial name Usibor ${ }^{\circledR} 1500 \mathrm{P}$ ) were fully austenitized in a furnace at over $900{ }^{\circ} \mathrm{C}$ and subjected to a carefully controlled cooling process, leading to fully martensitic, bainitic and ferritic/pearlitic micro-structures, respectively. In the subsequent sections, the three main grades of the investigated 22MnB5 material will be referred to as martensite (M) or "cooled tool sheet", bainite (B) or "heated tool sheet", and ferrite/pearlite (F) or "open furnace sheet".

A comprehensive test program on the strain hardening and fracture behavior of the main grades has been performed, where the deformation and fracture behavior is generalized by the introduction of hardness-based interpolation methods. The model has been integrated into the modular user material model frame work (MMM) developed at Volkswagen Group Research [1]. The present paper focusses on the development of the shell element extensions.

\section{Hardness-based necking/post-necking model}

An extended Swift law is introduced for fitting all three main grades using the same analytical function [1]. The modified Mohr-Coulomb fracture model (mMC) by Bai and Wierzbicki [2] is used in this work, which is based on the classical Mohr-Coulomb (MC) criterion [3, 4]. A review of the fracture model theory and the combined experimental/numerical model parameter identification procedure for the three main grades is provided in [1]. In FIGURE 1c, the mMC fracture curves for the main grades (thick solid lines) and some interpolated curves are shown.

2D finite elements (shells) cannot represent the 3D stress state beyond necking, since the emerging stress in the thickness direction is neglected. Using a refined shell mesh, the equivalent strains in the localized necked region increase dramatically due to the missing constraint by stress development in the thickness direction, e.g. [5, 6]. Hence, the fracture model introduced in the previous section can only be used for a shell model, when fracture occurs under plane stress conditions. In other words, the application of the mMC fracture model to shell elements is limited to non-necking load cases, such as surficial cracks under bending or in-plane shear-induced cracks.

For the sake of computational efficiency, vehicle crash simulation models usually consist of rather coarse shell elements of 3-5 mm length. For such a coarse mesh, the element size is larger than the localized necking zone, so 
that in contrast to a refined shell mesh, a coarse shell element mesh exhibits only moderate strain localization. This apparent drawback is actually beneficial for the modeling and will be exploited subsequently for the development of the post-necking model.

The widely known model by Marciniak and Kuczynski (MK) [7] for localized necking instability prediction has been applied, in order to determine the necking behavior of the three main material grades, FIGURE 1a. A full integration of the MK model into simulation codes is the most accurate way to predict the MK necking strains for arbitrary loading conditions. However, such an implementation can significantly increase the simulation time. Hence, for computational efficiency, the MK concept is applied to determine the necking strains for linear monotonic loading paths as a function of the load state using numerical finite element simulations, FIGURE 1 b. The finite element patch features an initial imperfection (reduced thickness zone) at an arbitrary angle of $\varphi$, FIGURE 1b. The initial imperfection is defined by $f_{0}=t_{B} / t_{A}$, where $t_{A}$ is the sheet thickness, and $t_{B}$ is the groove thickness. The global load state is defined by the ratio of principal plastic strains applied to the patch,

$$
\alpha=\frac{d \varepsilon_{2}}{d \varepsilon_{1}} .
$$

The simulation model can be evaluated at arbitrary loading states $(\alpha)$ and groove orientations $(\varphi)$. Parameter $f_{0}$ has been calibrated for the uniaxial tensile tests $(\alpha=-1 / 2)$ such that the state of localized necking, indicated by a strain bifurcation (plastic strain in zone B >> plastic strain in zone A) is reached at the state of maximum stress obtained in the corresponding tensile test. Now, the procedure is repeated for other distinct load states, so that the global equivalent plastic strain at necking, $\bar{\varepsilon}_{n}^{p}$, is determined numerically in the range $-1 / 2<\alpha \leq 1$ (see points in FIGURE 1c). In the next step, these virtual necking state points are represented by an empirical necking model in the MMM user subroutine which is applied to the central through-thickness integration point of the shell elements.

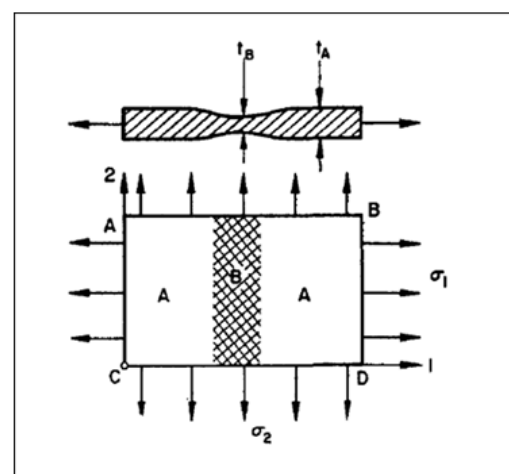

a)

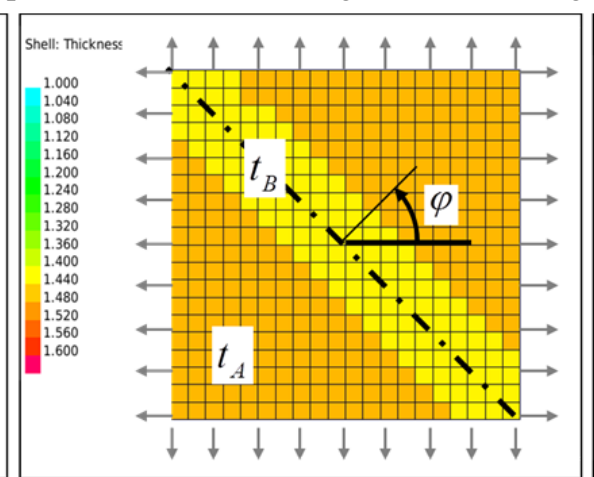

b)

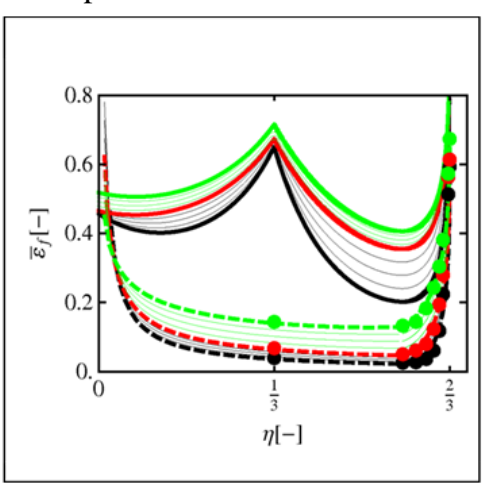

c)

FIGURE 1. (a) Original concept for localized necking instability prediction [7]; (b) finite element model of the MK concept; c) plane stress curves of the modified Mohr-Coulomb (mMC) fracture model (thick solid lines), generalized necking model curves (thick dashed lines) fitted through the virtual necking data points obtained from MK analysis (points), and hardness-based interpolation curves for the fracture and necking models (thin lines).

The classical necking models by Hill [8] and Stören-Rice [9] have been found inappropriate to accurately represent the necking behavior of the investigated material grades. Hence, the proposed generalized necking model is based on an extension of the necking model by Hill [8] for $-1<\alpha<0$ and introduces a pure empirical model for 0 $\leq \alpha \leq 1$. The equivalent necking strain of the model by Hill reads

$$
\bar{\varepsilon}_{n, \text { Hill }}=\frac{2 n \sqrt{1+\alpha+\alpha^{2}}}{\sqrt{3}(1+\alpha)} \quad \text { for }-1<\alpha \leq 0 \text {, }
$$

where $n$ is the hardening exponent of the underlying Swift law. The proposed two-branch generalized necking model reads 


$$
\bar{\varepsilon}_{n}=\left\{\begin{array}{cc}
\bar{\varepsilon}_{n, \text { Hill }}(1+\alpha)^{p}=\sqrt{1+\alpha+\alpha^{2}}(1+\alpha)^{p-1} \varepsilon_{P S T} & \text { for } \quad-1<\alpha \leq 0 \\
\varepsilon_{P S T}+\left(\varepsilon_{B T}-\varepsilon_{P S T}\right) \alpha^{m} & \text { for } 0<\alpha \leq 1
\end{array} .\right.
$$

In eqn (3), the flexibility of the classical Hill model is enhanced by adding another term, where $p$ can be substituted by the equivalent plastic necking strains under uniaxial tension, $\varepsilon_{U T}$, and plane strain tension, $\varepsilon_{P S T}$,

$$
p=\frac{\operatorname{Ln}(2)-\operatorname{Ln}\left(\frac{2}{\sqrt{3}} \frac{\varepsilon_{U T}}{\varepsilon_{P S T}}\right)}{\operatorname{Ln}(2)} .
$$

For positive principal strain ratios, an empirical equation is introduced in eqn (3), where $\varepsilon_{B T}$ is the equivalent plastic necking strain at equi-biaxial tension loading and $m$ is a shape parameter. Free parameters $\varepsilon_{U T}, \varepsilon_{P S T}, \varepsilon_{B T}$ and $m$ have to be determined from experimental data, where in the present study, the proposed necking model parameters are identified from the virtual data points obtained from the MK analysis (dashed lines in FIGURE 1c). In order to take into account non-proportional loading, an accumulative necking state formalism is introduced via

$$
D_{\text {neck }}=\int \frac{\Delta \bar{\varepsilon}^{p}}{\bar{\varepsilon}_{n}(\alpha)}
$$

where necking occurs at $D_{\text {neck }} \geq 1$.

For the post-necking behavior it is assumed that the shell mesh can at least represent moderate strain localization beyond necking. That is, considering a tensile test, the strain is assumed to localize in only a few element rows along the gage section after initiation of localized necking in the specimen. Since the shell element length is larger than the necking region, the post-necking deformation is only taking place in the shell elements of moderate strain localization, where the maximum post-necking deformation, $\Delta L_{\text {post }}$, is equal to a characteristic post-necking deformation length, $L_{c}$. The post-necking deformation is calculated by

$$
\Delta L_{\text {post }}=L_{@ \text { neck }}\left(e^{\Delta \bar{\varepsilon}_{\text {post }}^{p}}-1\right),
$$

where $L_{@ n e c k}$ is the element length at the state of necking in the direction of first principal strain. $\Delta \bar{\varepsilon}_{\text {post }}^{p}$ is the accumulated equivalent plastic post-necking strain. It will be shown subsequently that due to the length-based formulation the post-necking model is quite mesh-independent.

Generalized hardness-based necking model: Basically, our model implies a 2-phase decomposition of the material phases for continuous cooling of the material [1]. Depending on the material hardness, the material contains ferrite/pearlite plus bainite phases, or bainite plus martensite phases, where the "pure" phases (ferrite/pearlite, bainite, and martensite) represent the three main material grades characterized and modeled in the previous sections. The generalized hardness-based necking model is obtained by linear combination of the main grade necking curves:

$$
\bar{\varepsilon}_{n}(H V)=\left\{\begin{array}{lll}
X_{B} \bar{\varepsilon}_{n, B}+\left(1-X_{B}\right) \bar{\varepsilon}_{n, F} & \text { for } & H V_{F} \leq H V \leq H V_{B} \\
X_{B} \bar{\varepsilon}_{n, B}+\left(1-X_{B}\right) \bar{\varepsilon}_{n, M} & \text { for } & H V_{B}<H V \leq H V_{M}
\end{array}\right.
$$

with the bainite phase content

$$
X_{B}(H V)=\left\{\begin{array}{lll}
\frac{H V-H V_{F}}{H V_{B}-H V_{F}} & \text { for } & H V_{F} \leq H V \leq H V_{B} \\
\frac{H V-H V_{M}}{H V_{B}-H V_{M}} & \text { for } & H V_{B}<H V \leq H V_{M}
\end{array}\right.
$$

where $H V_{F}$ is the hardness of the pure ferrite/pearlite phase (open furnace sheets), $H V_{B}$ is the hardness of the pure bainite phase (heated tool sheets), and $H V_{M}$ is the hardness of the pure martensite phase (cooled tool sheets).

As a demonstration of the hardness-based necking model, the uniaxial tensile tests of the main grades and the intermediate grades are simulated using a $0.5 \mathrm{~mm}$ solid element model and a $5 \mathrm{~mm}$ shell element model. The 
predicted load-displacement curves of both model approaches are in very good agreement with the experiments, FIGURE 2, where the typical "banana-shape" relation of strength and elongation can be well recognized. It is interesting to note that due to the mesh-regulating nature of the post-necking model, shell element models using different mesh sizes (2mm, 5mm, 10mm) provide a similar load-displacement response, FIGURE 3.

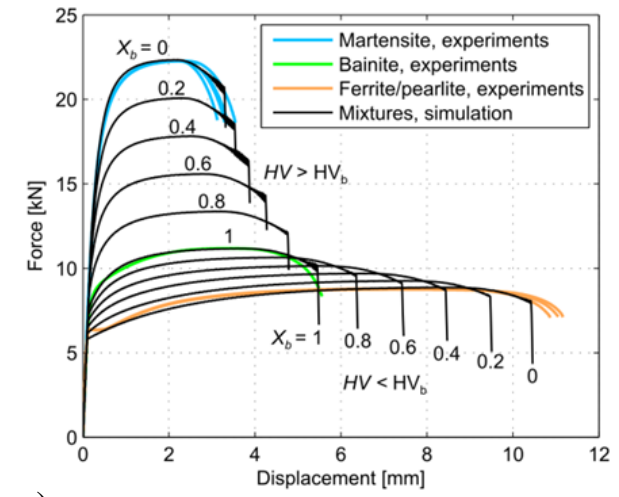

a)

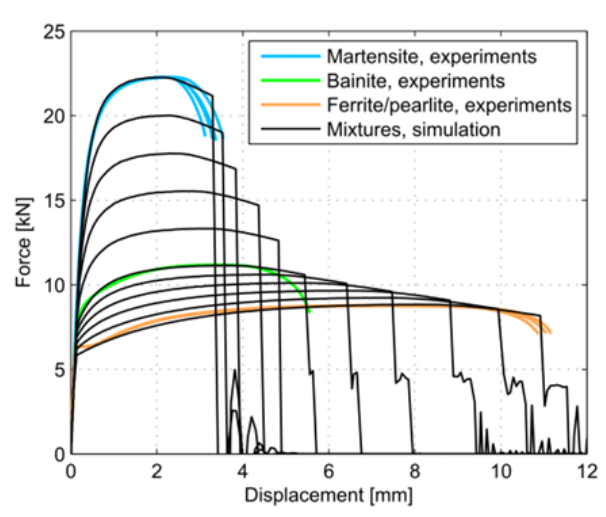

b)

FIGURE 2. Load-displacement curves of uniaxial tensile tests of the three main grades of $22 \mathrm{MnB} 5$ (colored) and corresponding simulations with a Vickers hardness (HV) variation (black): (a) 0.5 mm solid element mesh; (b) 5 mm shell mesh.

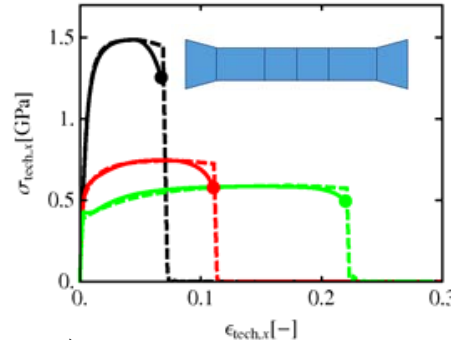

a)

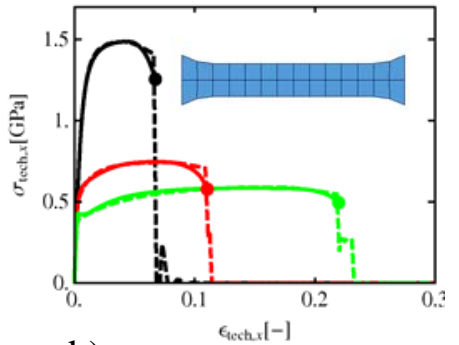

b)

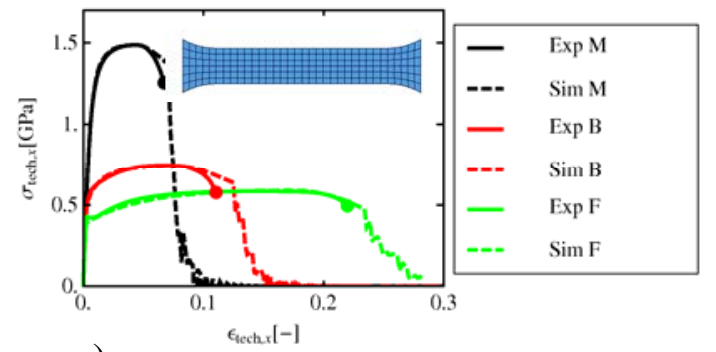

c)

FIGURE 3. Engineering stress-strain curves of uniaxial tensile tests of the three main grades and simulations using different mesh sizes: (a) $10 \mathrm{~mm}$ mesh size; (b) $5 \mathrm{~mm}$ mesh size; (c) $2 \mathrm{~mm}$ mesh size.

\section{REFERENCES}

1. T. K. Eller, L. Greve, M. T. Andres, M. Medricky, A. Hatscher, V. T. Meinders and T. van den Boogaard, "Plasticity and fracture modeling of quench-hardenable boron steel with tailored properties", submitted to the Journal of Materials Processing Technology, May 2013

2. Y. Bai and T. Wierzbicki, Int. J. Frac., 169, 1-20 (2010)

3. C. Coulomb, Mem Acad Roy des Sci, (1776)

4. O. Mohr, „Abhandlungen aus dem Gebiete der Technischen Mechanik“, 2nd ed., Ernst, Berlin (1914)

5. D. Mohr and F. Ebnoether, Int. J. Solids Struct.,46(20), 3535-3547 (2009)

6. L. Greve, „Simulation des Bruchverhaltens mit automatischer lokaler Netzverfeinerung“, VDI-Berichte Nr. 2107, 2010, pp. 169-181

7. Z. Marciniak and K. Kuczynski, Int. J. Mech. Sci., 9(9), 609-612 (1967)

8. R. Hill, J. Mech. Phys. Solids, 1(1), 19-30 (1952)

9. S. Storen and J. R. Rice, J. Mech. Phys. Solids, 23(6), 421-441 (1975) 